\title{
Synthetic antimicrobial peptide tuning permits membrane disruption and interpeptide synergy
}

Authors: Francisco R. Fields ${ }^{1,2,3}$, Giorgia Manzo ${ }^{4}$, Charlotte K. Hind ${ }^{5}$, Jeshina Janardhanan ${ }^{6}$, Ilona P. Foik ${ }^{7}$, Phoebe Do Carmo Silva ${ }^{5}$, Rashna D. Balsara ${ }^{6,9}$, Melanie Clifford ${ }^{5}$, Henry M. $\mathrm{Vu}^{1,9}$, Jessica N. Ross ${ }^{1,2}$,Veronica R. Kalwajtys ${ }^{1}$, Alejandro J. Gonzalez ${ }^{1}$, Tam T. Bui ${ }^{10}$, Victoria A. Ploplis ${ }^{6,9}$, Francis J. Castellino ${ }^{6,9}$, Albert Siryaporn ${ }^{7,8}$, Mayland Chang, ${ }^{3,6}$, J. Mark Sutton ${ }^{5}$, A. James Mason ${ }^{4}$, Shaun Lee ${ }^{1,2,3, *}$

\section{Affiliations:}

${ }^{1}$ Department of Biology, University of Notre Dame, Notre Dame, IN 46556

${ }^{2}$ Eck Institute of Global Health, University of Notre Dame, Notre Dame, IN 46556

${ }^{3}$ Chemistry Biology Biochemistry Interface, University of Notre Dame, Notre Dame, IN 46556

${ }^{4}$ Institue of Pharmaceutical Science, School of Cancer \& Pharmaceutical Science, King's College London, Franklin-Wilkins Building, 150 Stamford Street, London, SE1 9NH, United Kingdom

${ }^{5}$ Technology Development Group, National Infection Service, Public Health England, Salisbury, UK

${ }^{6}$ Department of Chemistry and Biochemistry, University of Notre Dame, Notre Dame, IN 46556

${ }^{7}$ Department of Physics and Astronomy, University of California Irvine, Irvine, CA 92697

${ }^{8}$ Department of Molecular Biology and Biochemistry, University of California Irvine, Irvine CA 92697

${ }^{9}$ W.M Keck Center for Transgene Research, University of Notre Dame, Notre Dame, IN 46556

${ }^{10}$ Centre for Biomolecular Spectroscopy and Randall Division of Cell and Molecular Biophysics, King's College London, New Hunt's House, London, SE1 1UL, United Kingdom

*1ee.310@nd.edu

Correspondence to: lee.310@nd.edu

This PDF file includes:

Materials and Methods

Supplemental Figures 1-6 
Supplemental Tables 1-4 


\section{Materials and Methods}

\section{Peptides and Lipids}

Peptides were commercially synthesized by Genscript (Piscataway, NJ). All peptides were synthesized to $>95 \%$ purity and verified by HPLC and mass spectrometry. Peptide sequences for this study are found in supplemental figure 1. Design strategy for syn-safencin and its variants is found in our previous work ${ }^{10}$. The lipids 1-palmitoyl-2-oleoyl-sn-glycero-3phospho-(1'-rac-glycerol) (POPG), 1-palmitoyl-2-oleoyl-sn-glycero-3-phosphoethano- lamine (POPE), 1,2-diphytanoyl-sn-glycero-3-phospho-(1'-rac- glycerol) (DPhPG) and 1,2-diphytanoylsn-glycero-3-phosphoethanolamine (DPhPE) were purchased from Avanti Polar Lipids, Inc.

(Alabaster, AL).

\section{Peptide Cytotoxicity Assays}

Eukaryotic cytotoxicity was determined by ethidium homodimer as described previously ${ }^{10}$. Ethidium homodimer assays were carried out with HaCaT cells in 24 well culture dishes grown to $90 \%$ confluency. Medium was aspirated and cells were washed with PBS. Peptides were resuspended in 100\% DMEM and added to the cells at the desired concentration. Cells were incubated with peptide for 16 hours. Medium was aspirated and cells were washed three times with PBS. Cells were incubated in $4 \mu \mathrm{M}$ ethidium homodimer (Molecular Probes) in PBS for 30 minutes. Level of fluorescence was determined by 528 excitation and $617 \mathrm{~nm}$ emission and a cutoff value of $590 \mathrm{~nm}$. Saponin (.1\%) was then added to each well and incubated for 20 minutes. The fluorescence was read again. Percent membrane permeabilization was determined by dividing the initial fluorescence by the second fluorescence reading.

Peptide Hemolysis Assays

For hemolysis assays, $1 \mathrm{~mL}$ of sheep red blood cells (RBCs) was washed 3 times in cold PBS. Washed cells were resuspended in $25 \mathrm{ml}$ of PBS. Triton, PBS, or peptide in PBS were added to $180 \mu \mathrm{L}$ of resuspended RBCs and incubated at 37C for 1 hour. Samples were read at $450 \mathrm{~nm}$. Data was expressed as percent hemolysis by relativizing to the Triton and PBS controls. Hemolysis assays were done in triplicate.

FACS

Overnight cultures of BL-21 E. coli were diluted to a starting OD $=1.1 \mathrm{~mL}$ of cells was washed 3 times in saline solution. Cells were then resuspended in $1 \mathrm{~mL}$ of saline, $70 \%$ isopropyl alcohol, or peptide $(20 \mu \mathrm{M})$ in saline and incubated at room temperature for 30 minutes. Cells were pelleted and resuspended in $1 \mathrm{~mL}$ of saline with $1.5 \mu \mathrm{L} / \mathrm{mL}$ propidium iodide (ThermoFischer) and incubated at room temperature in the dark for fifteen minutes. Samples were pelleted and washed three times with saline. Cells were suspended in $200 \mu \mathrm{L}$ of saline just prior to FACS loading. Samples were run on the FACs Aria (BD Biosciences) using the Texas Red channel. For synergy experiments, $5 \mu \mathrm{M}$ of syn-safencin 8 or 96 was used.

\section{GFP leakage}

Overnight cultures of GFP expressing BL-21 E.coli were diluted to a CFU of $5^{*} 10^{8}$ in PBS. $2 \mathrm{ml}$ of the cell suspension with $10 \mu \mathrm{M}$ peptide was imaged using a 60x objective oil immersion lens on a Nikon Eclipse Ti-E. FITC and DIC images were acquired every 10 minutes for 8 hours. Images were processed using Image $\mathrm{J}$.

MIC and FICI Assays

The MIC of the nine syn-safencin peptides were determined using a two-fold broth microdilution assay. Peptide stocks were diluted two-fold in non-cation adjusted Mueller-Hinton Broth within a 96 well polypropylene plate. Next, $100 \mu \mathrm{l}$ of bacterial suspension at $\mathrm{OD}=.01$ diluted from overnight cultures were added to the wells. The plate was incubated at $37^{\circ} \mathrm{C}$ for 18 
hours without shaking and the MIC was determined as the lowest concentration where no visible growth could be detected by measuring the absorbance (A600) on an Omega plate reader. FICI assays were carried out similarly to the MIC assay using a modified two-fold broth microdilution in non-cation adjusted Mueller-Hinton broth. FICI was calculated using the following equation:

$$
\text { FICI }=\frac{\text { MIC of compound A \& B combined }}{\text { MIC of compound A alone }}+\frac{\text { MIC of compound A \& B combined }}{\text { MIC of compound B alone }}
$$

FICI values $\leq .5$ were considered synergistic.

Liposome preparation.

Small unilamellar vesicles (SUVs) were prepared for circular dichroism (CD). Lipid powders were solubilized in chloroform and dried under rotor-evaporation. To completely remove the organic solvent, the lipid films were left overnight under vacuum and hydrated in 5 $\mathrm{mM}$ Tris buffer ( $\mathrm{pH}$ 7.0). Lipid suspension was subjected to 5 rapid freeze-thaw cycles for further sample homogenization. POPE/POPG (75:25, mol:mol) and POPG SUVs were obtained by sonicating the lipid suspension on Soniprep 150 (Measuring and Scientific Equipment, London, UK) for $3 \times 7$ minutes with amplitude of 6 microns in the presence of ice to avoid lipid degradation. The SUVs were stored at $4{ }^{\circ} \mathrm{C}$ and used within 5 days of preparation.

Circular Dichroism Spectroscopy

Far-UV spectra of the peptides in the presence of SUVs and SDS micelles were acquired on a Chirascan Plus spectrometer (Applied Photophysics, Leatherhead, UK). Liposome samples were maintained at $310 \mathrm{~K}$. Spectra were recorded from 260 to $190 \mathrm{~nm}$ in a $0.5 \mathrm{~mm}$ cuvette. Lipid suspension was added to the cuvette at a final concentration of $5.0 \mathrm{mM}$ and then a few $\mu \mathrm{l}$ of a concentrated peptide solution was added and thoroughly mixed to give the indicated final peptide-to-lipid molar ratios. In processing, a spectrum of the peptide free suspension was subtracted and Savitsky-Golay smoothing with a convolution width of 5 points applied. NMR Spectroscopy

Liquid state NMR spectroscopy experiments and data analysis were performed as described previously $18,19,25$. The sample solution consisted of $500 \mu \mathrm{M}$ peptide and $50 \mathrm{mM}$ deuterated sodium dodecyl sulphate (SDS-d25) micelles. 10\% D2O containing trimethylsilyl propanoic acid (TSP) was added for the lock signal and as internal chemical shift reference. NMR spectra were acquired on a Bruker Avance $500 \mathrm{MHz}$ spectrometer (Bruker, Coventry, UK) equipped with a cryoprobe. Standard Bruker TOCSY and NOESY pulse sequences were used, with water suppression using an excitation sculping sequence with gradients. The $1 \mathrm{H} 90$-degree pulse length was $8.0 \mathrm{~s}$. Two TOCSY with mixing times of 60 and $80 \mathrm{~ms}$, and two NOESY with mixing time of 100 and $200 \mathrm{~ms}$ were acquired for each sample. The relaxation delay was $1 \mathrm{~s}$. 2048 data points were recorded in the direct dimension, and either 256 or 512 data points in the indirect dimension. Dynamo software was used for the structure calculation. Inter-proton NOEs interactions were used as distance restraints in the structure calculation. 1000 structures were generated using Dynamo's annealing protocol. Only unambiguous NOEs were used in this case after being classified as strong, medium and weak on the base of the relative intensity of the cross-peaks in the NOESY spectra. On the base of this classification an upper limit of $0.27,0.33$ and $0.50 \mathrm{~nm}$ have been applied, respectively, as restraint on the corresponding inter-proton distance, as described previously. One thousand structures were calculated and the 100 conformers with the lowest potential energy were selected for the analysis. The selected 100 conformers were aligned, and the root mean square deviation (RMSD) of the backbone heavy atoms was calculate with respect to their average structure. Solvent molecules were not included in the calculations. Structural coordinates were deposited in the Protein Data Bank 
(www.rcsb.org) 608J, 608P, 608R, 608S, and 608T for syn-safencin 1, 8, 24, 56, and 96 respectively

Electrophysiology experiments (Patch-clamp)

Patch-clamp experiments were done as described previously ${ }^{18,19}$. Giant unilamellar vesicles (GUVs) composed of DPhPE/DPhPG (60:40, mol:mol) and DPhPG were prepared in the presence of $1 \mathrm{M}$ sorbitol by the electroformation method in an indium-tin oxide (ITO) coated glass chamber connected to the Nanion Vesicle Prep Pro setup (Nanion Technologies GmbH, Munich, Germany) using a 3-V peak-to-peak AC voltage at a frequency of $5 \mathrm{~Hz}$ for 120 and 140 minutes, respectively, at $36^{\circ} \mathrm{C}$. Bilayers were formed by adding the GUVs solution to a buffer containing $250 \mathrm{mM} \mathrm{KCl}, 50 \mathrm{mM} \mathrm{MgCl} 2$ and $10 \mathrm{mM}$ Hepes (pH 7.00) onto an aperture in a borosilicate chip (Port-a-Patch ${ }^{\circledR}$; Nanion Technologies) and applying 70-90 mbar negative pressure resulting in a solvent-free membrane with a resistance in the $\mathrm{G} \Omega$ range. After formation, a small amount of peptide stock solution (in water) was added to $50 \mu \mathrm{L}$ of buffer solution in order to obtain a concentration of $40 \mu \mathrm{M}$. For synergy assessment, peptides were used at a final concentration of $10 \mu \mathrm{M}$. All the experiments were carried on with a positive holding potential of $50 \mathrm{mV}$. For all the experiments a minimum of 6 according repeats was done. Current traces were recorded at a sampling rate of $50 \mathrm{kHz}$ using an EPC-10 amplifier from HEKA Elektronik (Lambrecht, Germany). The system was computer controlled by the PatchControl ${ }^{\mathrm{TM}}$ software (Nanion) and GePulse (Michael Pusch, Genoa, Italy,

http://www.ge.cnr.it/ICB/conti_moran_pusch/programs-pusch/software-mik.htm). The data were filtered using the built-in Bessel filter of the EPC-10 at a cut-off frequency of $10 \mathrm{kHz}$. The experiments were performed at room temperature. Data analysis was performed with the pClamp 10 software package (Axon Instruments). Estimation of pore radii was performed as previously ${ }^{20}$.

Transmission electron microscopy

$P$. aeruginosa PAO1 was cultured in in LB-Miller medium (Beckton, Dickinson, Franklin Lakes, $\mathrm{NJ}$ ) overnight to saturation at $37^{\circ} \mathrm{C}$ in a roller drum at $100 \mathrm{rpm}$, centrifuged at $3400 \mathrm{~g}$ for $5 \mathrm{~min}$, and resuspended in $0.85 \%(\mathrm{w} / \mathrm{v})$ sodium chloride. The suspension was diluted to an optical density of 1 as measured by absorbance at $600 \mathrm{~nm}$, treated with $20 \mu \mathrm{M}$ SynSaf-P96, $20 \mu \mathrm{M}$ SynSaf-P8, or a combination of $5 \boldsymbol{\mu}$ M SynSaf-P8 with $5 \boldsymbol{\mu}$ M SynSaf-P96 or $2 \boldsymbol{\mu}$ M SynSaf-P8 with $2 \boldsymbol{\mu} \mathrm{M}$ SynSaf-P96. After incubation at room temperature for $30 \mathrm{~min}$, Formvar/Carboncoated copper grids (200 mesh, Electron Microscopy Sciences, USA) were placed on bacterial suspension droplets and stained with 1\% uranyl acetate. Grids were imaged using a JEOL JEM2100F TEM (JEOL USA Inc., Peabody, MA), an accelerating voltage of $200 \mathrm{kV}$, emission currents between 102-126 $\boldsymbol{\mu} \mathrm{A}$, and using a Gatan OneView Camera (Gatan Inc., Pleasanton, CA, USA) using $4 \mathrm{~K} \times$ $4 \mathrm{~K}$ resolution.

Galleria mellonella in vivo infection studies

G. mellonella larvae (200-300 mg body weight) at the final instar stage were obtained from Speedyworm (Alexandria, MN). Meropenem was purchased from Sigma-Aldrich (St. Louis, MO). In order to determine the inoculum dose for the study, 10 larvae per group were injected with 5fold dilutions of Acinetobacter baumannii AYE strain ranging from $10^{5}$ to $10^{8} \mathrm{CFU}$ per larva. A $25-\mu \mathrm{L}$ Hamilton syringe was used to inoculate $10 \mu \mathrm{L}$ of the bacterial suspension into the hemocoel of the larvae through the last left proleg. Following infection, the larvae were housed in plastic petri dishes and incubated at $37^{\circ} \mathrm{C}$ in the dark. Larvae were monitored for survival every day for 3 days; dead larvae appear black in color and are unresponsive to touch. Peptides were dissolved in PBS to a concentration of $3.75 \mathrm{mg} / \mathrm{mL}$ and $10 \mu \mathrm{L}$ (equivalent to a dose of $150 \mathrm{mg} / \mathrm{kg}$ ) was 
injected into healthy uninfected larvae ( $\mathrm{n}=10$ per group) to assess peptide toxicity. Survival was recorded every day for 3 days. The surface of the larvae was swabbed with $70 \%$ ethanol with cotton Q-tips. Larvae were injected with $10 \mu \mathrm{L}$ of Acinetobacter baumannii AYE strain suspension to a final concentration of $10^{6} \mathrm{CFU}$ per larva. One hour after infection, larvae were treated with peptides at $100 \mathrm{mg} / \mathrm{kg}$, meropenem (positive control) at $60 \mathrm{mg} / \mathrm{kg}$ or PBS (negative control). For synergy assessment, peptides were injected as single formulations at $25 \mathrm{mg} / \mathrm{kg}$ or as a dual combination of $25 \mathrm{mg} / \mathrm{kg}$ of each peptide. The peptides and meropenem were dissolved in PBS and sterilized by filtration through $0.2 \mu \mathrm{m}$ nylon membrane (Pall Life Sciences, Port Washington, NY). The larvae were observed for 3 days to check for mortality. The larvae were considered dead if they were black in appearance and unresponsive to touch. The experiment was conducted twice, and statistics completed using GraphPad Prizm. 
A.

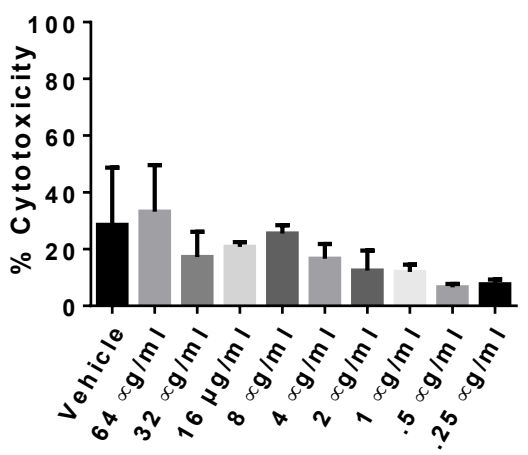

D.

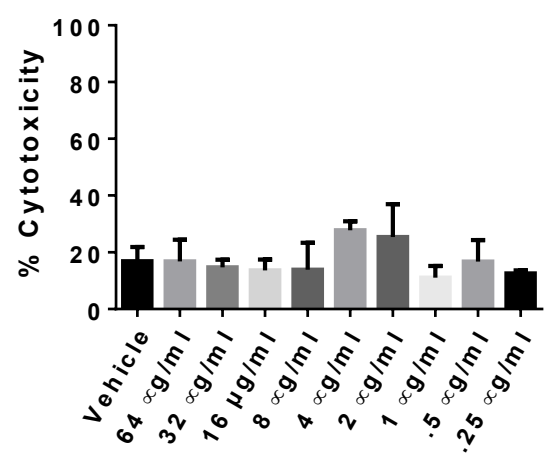

C.
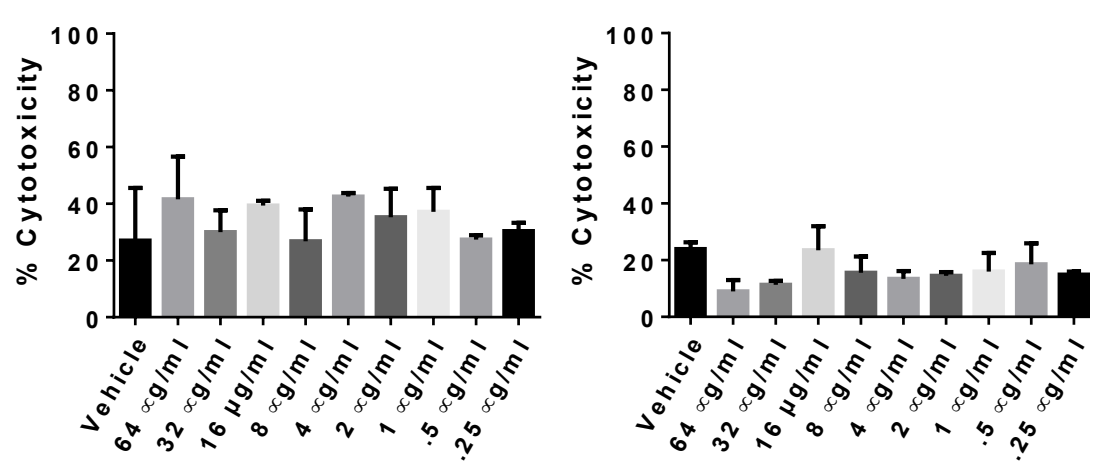

E.

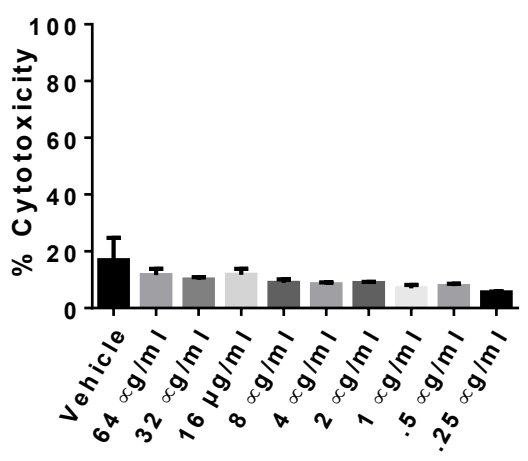

Figure S-1. Syn-safencin peptides exhibit no cytotoxicity on human keratinocytes up to 64 $\mu \mathrm{g} / \mathrm{mL}$. Ethidium homodimer cytotoxicity assays for SynSaf-P A. 1 B. 8 C. 24 D. 56 and E. 96 . All toxicity values were below $30 \%$ up to $64 \mu \mathrm{g} / \mathrm{mL}$ Data is representative of 3 technical replicates. Error bars indicate the standard deviation. 
A.

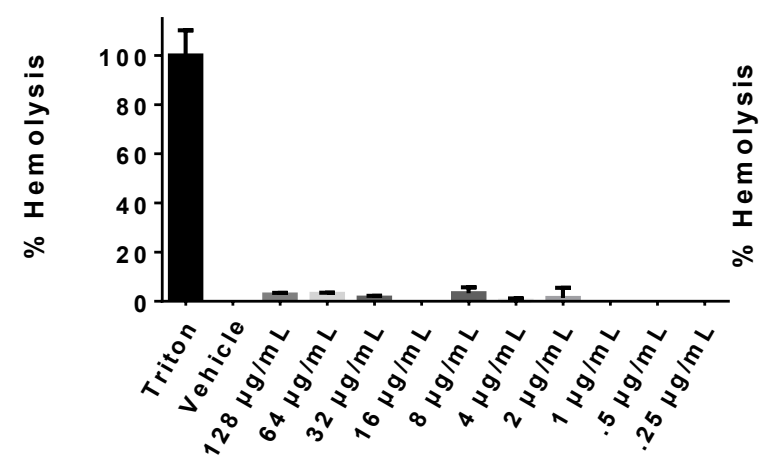

D.

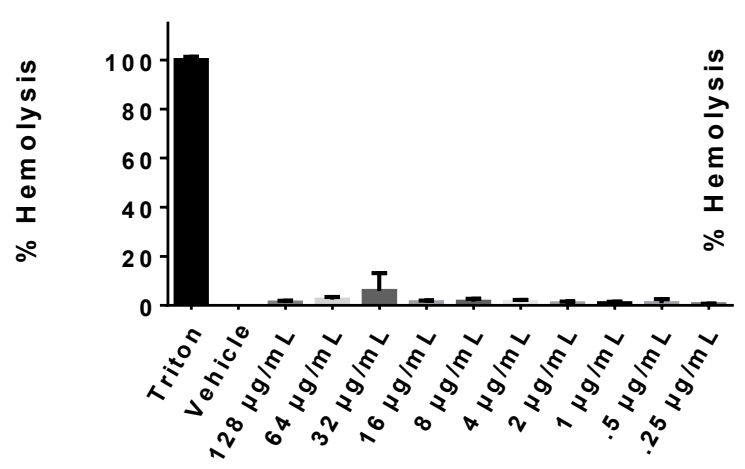

B.

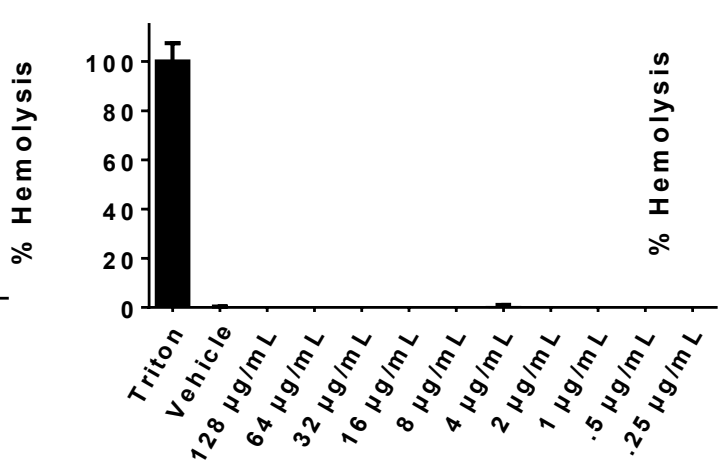

E.

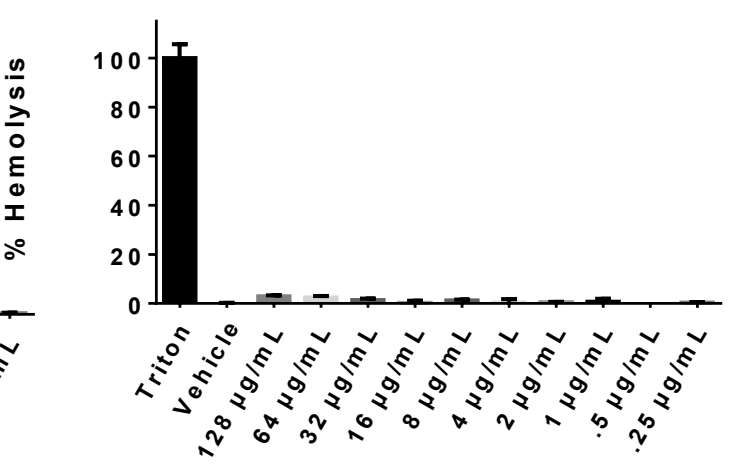

Figure S-2. Syn-safencin peptides are not hemolytic up to $128 \mu \mathrm{g} / \mathrm{mL}$. Hemolysis assays for SynSaf-P A. 1 B. 8 C. 24 D. 56 and E. 96 . All hemolysis values, relative to the Triton and vehicle controls, were below $10 \%$ up to $128 \mu \mathrm{g} / \mathrm{mL}$ Data is representative of 3 technical replicates. Error bars indicate the standard deviation. 


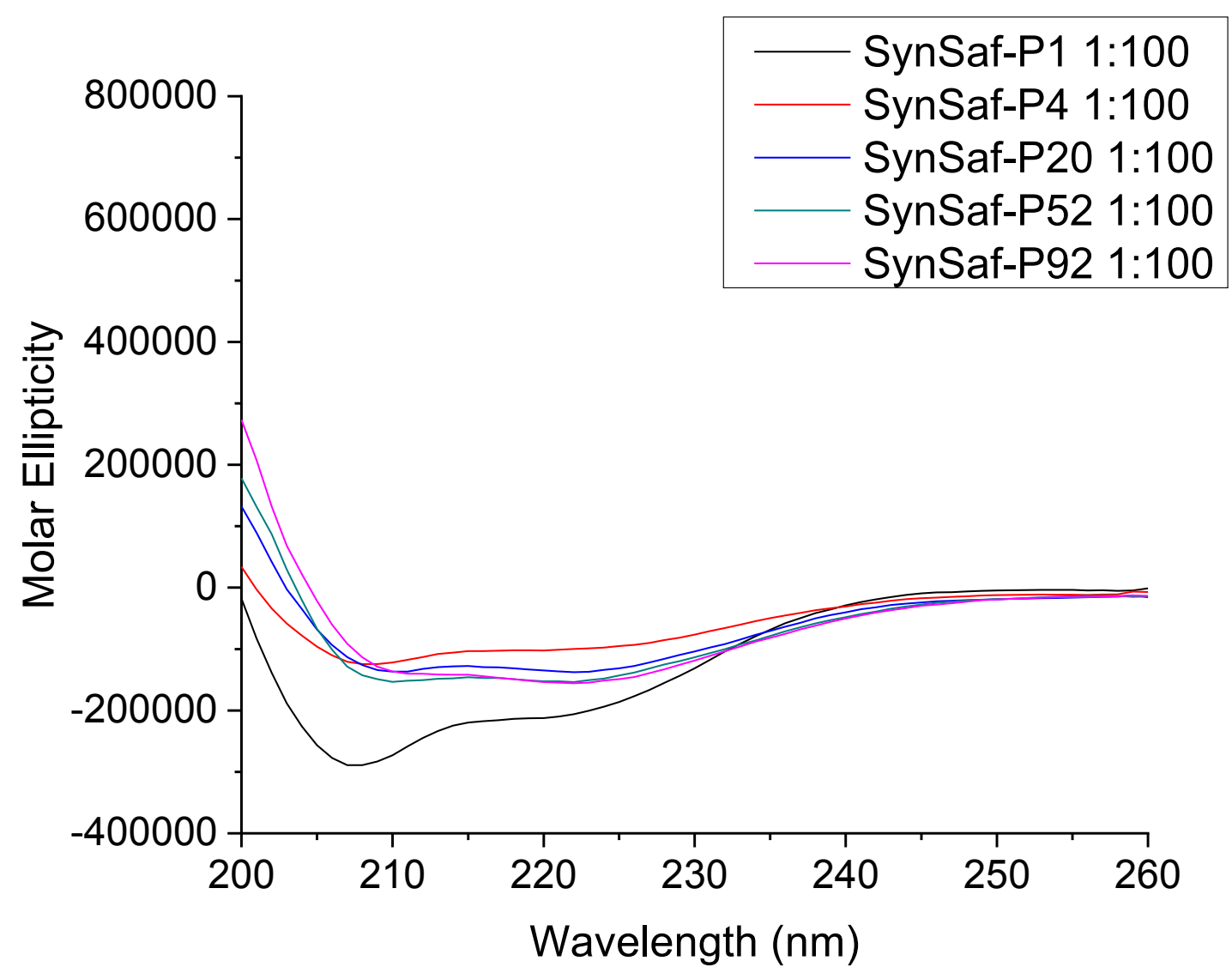

Figure S-3. Syn-safencin peptides adopt a helical conformation in a Gram-positive membranelike environments. All of the syn-safencin peptides adopt a helical conformation at a 1:100 peptide to lipid molar ratio in POPG micelles. 

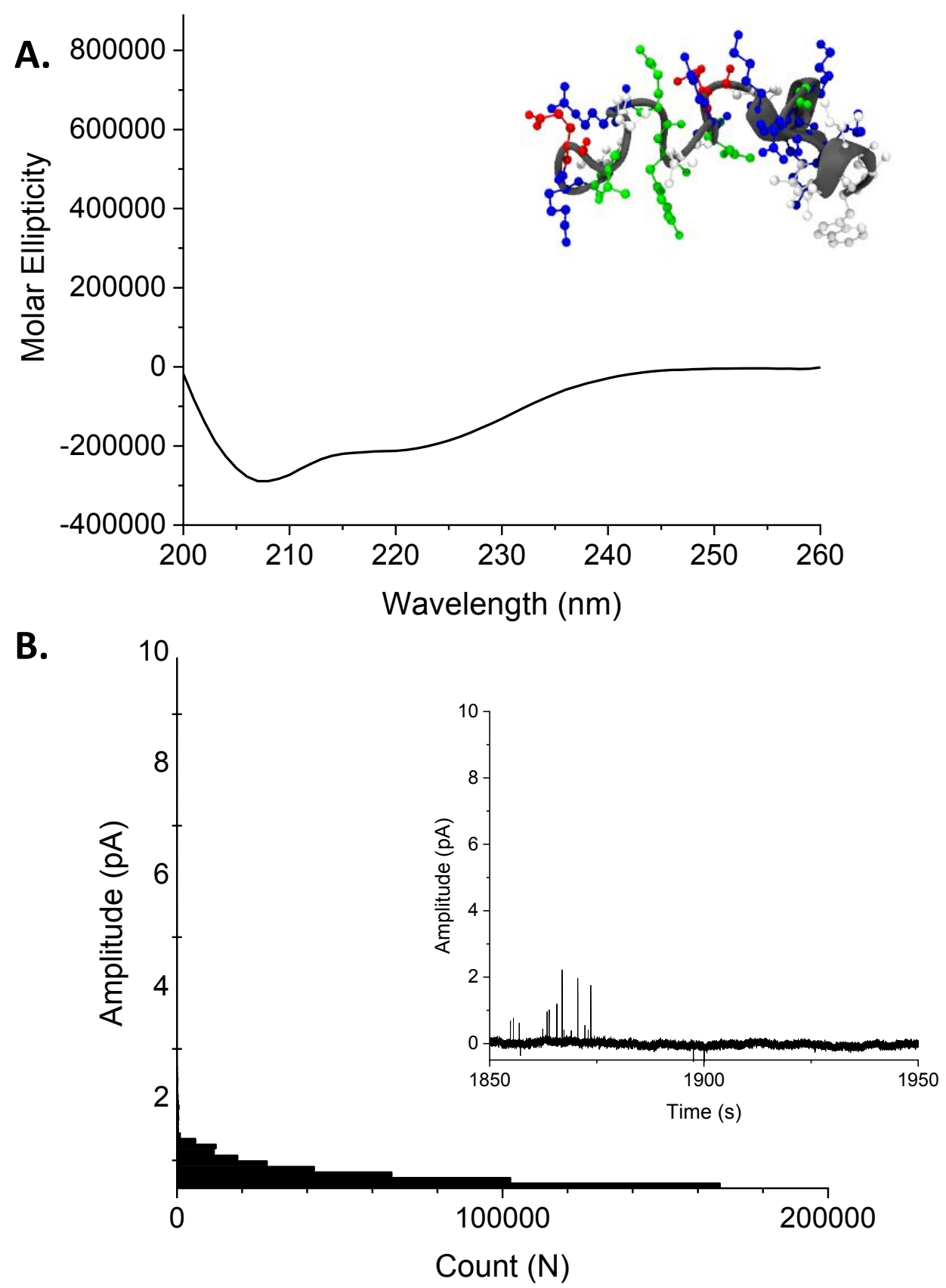

Figure S-4. Syn-safencin (SynSaf-P1) is a helical, non-membrane disrupting peptide. A) Secondary structure interrogation of SynSaf-P1 indicates that it is a helical peptide. CD data was collected in the presence of POPE:POPG SUVs. 2-D NMR structure of SynSaf-P1 determined in SDS micelles (inset). PDB accession number 608J. Basic, acidic, polar, and hydrophobic residues are in blue, red, green, and white respectively. B) SynSaf-P1 does not disrupt Gram negative model membranes, at $40 \mu \mathrm{M}$, as indicated by patch clamp analysis. All data point histogram and example trace (inset) of SynSaf-P24 are representative of six experiments $(n=6)$ done on DPhPE:DPhPG bilayers. 


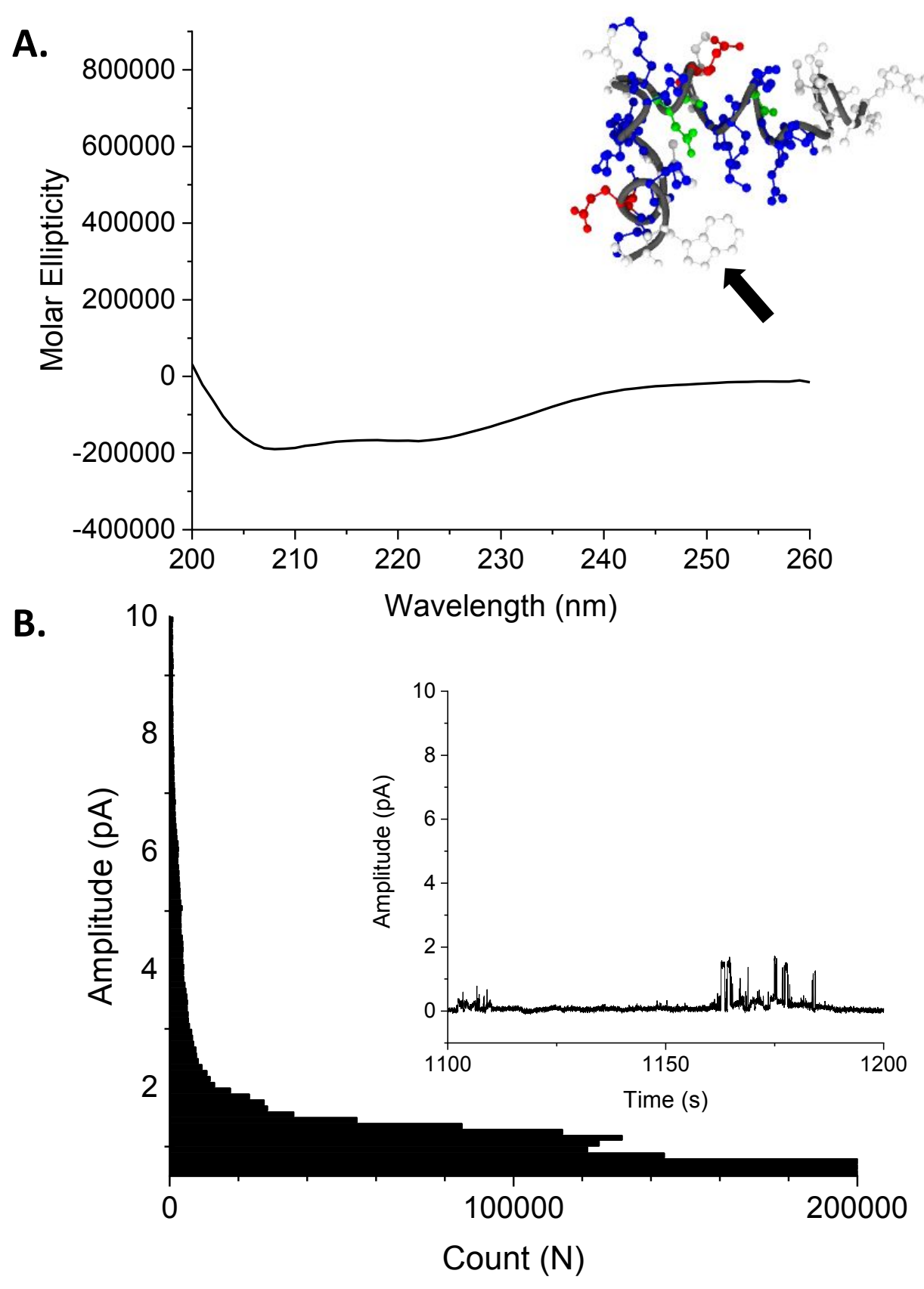

Figure S-5. Syn-safencin 24 (SynSaf-P24) is a helical, pore forming peptide. A) Secondary structure interrogation of SynSaf-P24 indicates that it is a helical peptide. CD data was collected in the presence of POPE:POPG SUVs. 2-D NMR structure of SynSaf-P24 determined in SDS micelles (inset). PDB accession number 608R. Arrows indicates the G2W substitution in Table 1. Basic, acidic, polar, and hydrophobic residues are in blue, red, green, and white respectively. B) SynSaf-P24 forms discrete pores on Gram negative model membranes, at $40 \mu \mathrm{M}$, as indicated by patch clamp analysis. All data point histogram and example trace (inset) of SynSaf-P24 are representative of six experiments $(n=6)$ done on DPhPE:DPhPG bilayers. 

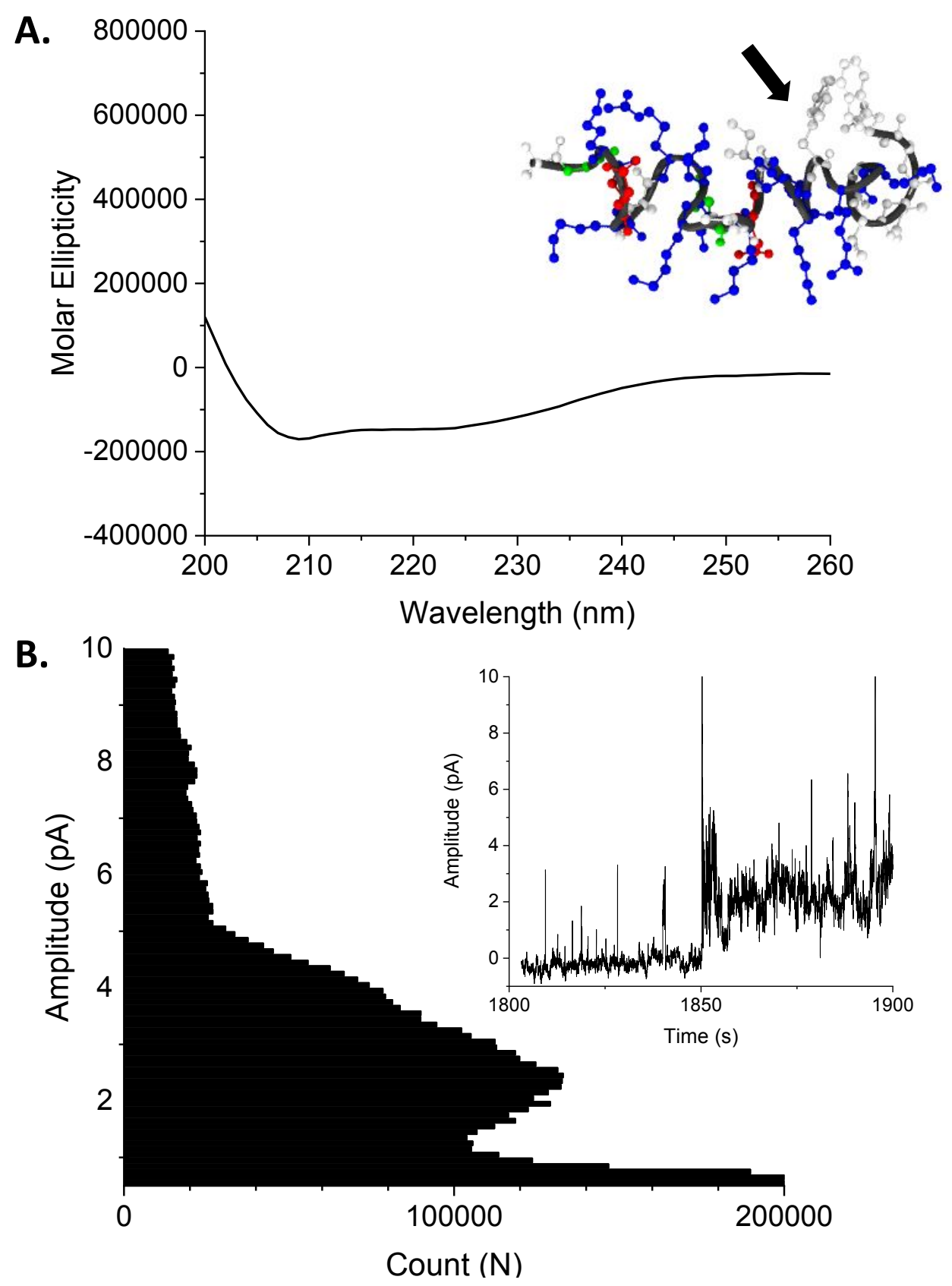

Figure S-6. Syn-safencin 56 (SynSaf-P56) is a helical, membrane disrupting peptide. A) Secondary structure interrogation of SynSaf-P56 indicates that it is a helical peptide. CD data was collected in the presence of POPE:POPG SUVs. 2-D NMR structure of SynSaf-P56 determined in SDS micelles (inset). PDB accession number 608S. Arrows indicates the G18W substitution in Table 1. Basic, acidic, polar, and hydrophobic residues are in blue, red, green, and white respectively. B) SynSaf-P56 causes large disruptions in Gram negative model membranes, at $40 \mu \mathrm{M}$, as indicated by patch clamp analysis. All data point histogram and example trace (inset) of SynSaf-P56 are representative of six experiments $(n=6)$ done on DPhPE:DPhPG bilayers. 


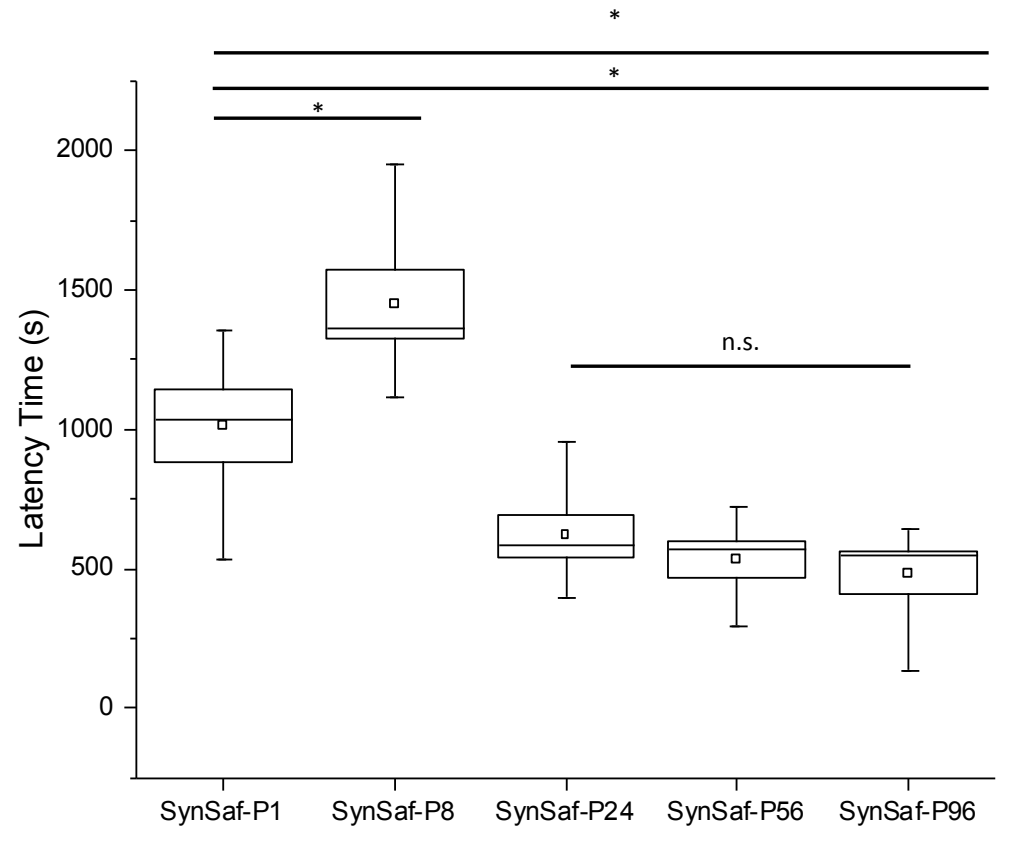

Figure S-7. W position does not significantly alter the time to membrane disruption. Latency times of the SynSaf-P96 series. A * indicates $\mathrm{p}<.05$ as determined by ANOVA and Tukey HSD post-hoc analysis $(\mathrm{n}=6)$. Error bars represent the range of the latency times. 


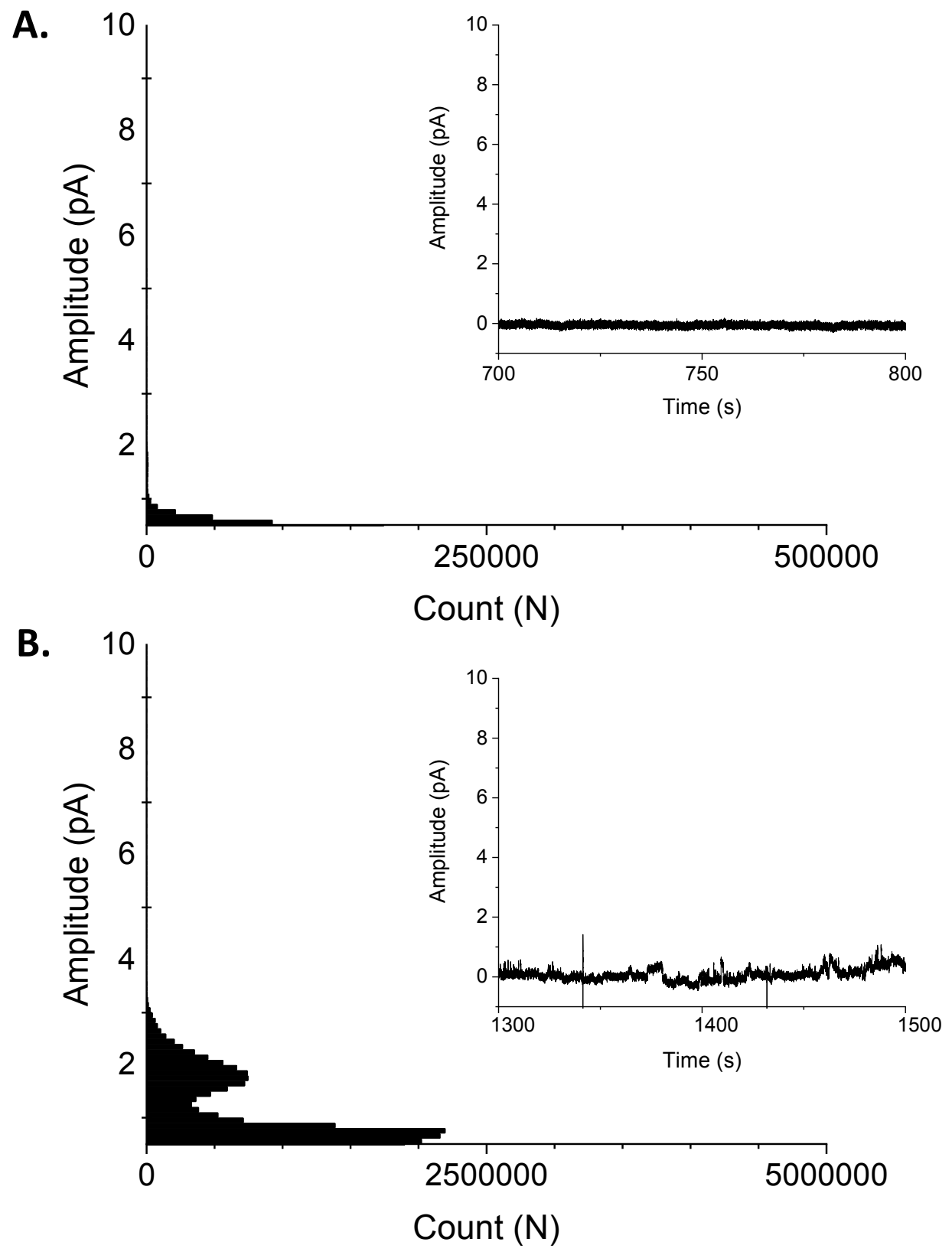

Figure S-8. SynSaf-P8 and SynSaf-P96 have reduced membrane disrupting activity at a reduced concentration. A) SynSaf-P8 does not disrupt Gram negative model membranes, at $10 \mu \mathrm{M}$, as indicated by patch clamp analysis. B) SynSaf-P96 only forms two populations of events on Gram negative model membranes, at $10 \mu \mathrm{M}$, as indicated by patch clamp analysis. All data point histograms and example traces (inset) of the each peptide are representative of six experiments $(n=6)$ done on DPhPE:DPhPG bilayers. 


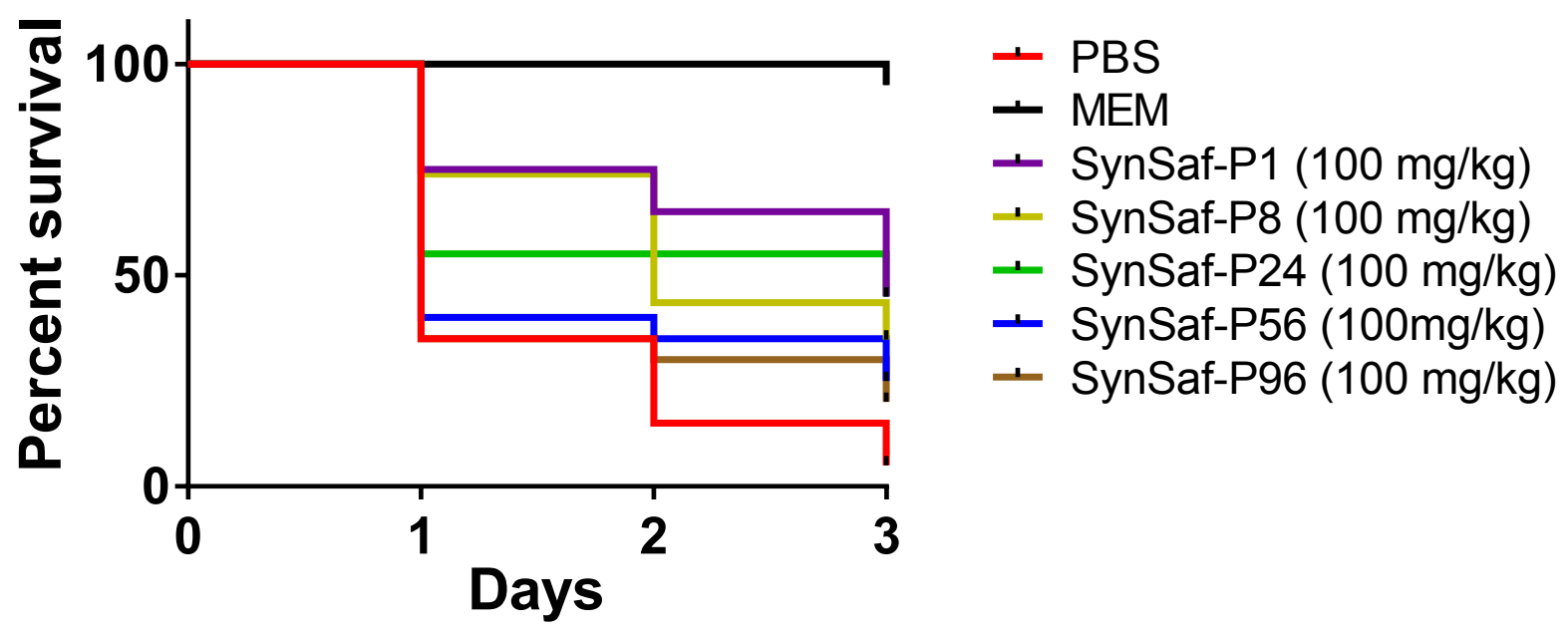

Figure S-9. In vivo evaluation of syn-safencin peptides using A. baumannii AYE infected $G$. mellonella larvae. 


\begin{tabular}{|c|c|c|c|c|c|}
\hline \multicolumn{6}{|c|}{ MIC Gram (-) panel $(\mu \mathrm{g} / \mathrm{ml})$} \\
\hline Strain & SynSaf-P1 & SynSaf-P8 & SynSaf-P24 & SynSaf-P56 & SynSaf-P96 \\
\hline K. pneumoniae NCTC 13368 & $>128$ & $>128$ & $>128$ & 128 & 16 \\
\hline K. pneumoniae M6 & $>128$ & $>128$ & $>128$ & 64 & 8 \\
\hline A. baumanii AYE & 32 & 64 & 8 & 2 & 1 \\
\hline A. baumanii ATCC 17987 & 64 & 64 & 16 & 8 & 1 \\
\hline P. aeruginosa PAO1 & $>128$ & $>128$ & $>128$ & 128 & 16 \\
\hline P. aeruginosa NCTC 13437 & $>128$ & $>128$ & $>128$ & $>128$ & 128 \\
\hline E. coli NCTC 12923 & 64 & 32 & 8 & 2 & 0.5 \\
\hline$X$. axonopodis pv. phaseoli & 4 & 1 & 0.5 & 0.5 & 0.25 \\
\hline P. syringae $\mathrm{pv}$. tomato & 16 & 4 & 0.5 & 1 & 0.25 \\
\hline
\end{tabular}

Table S-1. Minimal Inhibitory Concentration (MIC) values for the SynSaf-P96 series in $\mu \mathrm{g} / \mathrm{mL}$ on a panel of Gram-negative organisms. MIC is determined as the lowest concentration of peptide needed to prevent overnight growth. 


\begin{tabular}{|c|c|c|c|c|c|}
\hline \multicolumn{6}{|c|}{ MIC Gram (+) panel $(\mu \mathrm{g} / \mathrm{ml})$} \\
\hline Strain & SynSaf-P1 & SynSaf-P8 & SynSaf-P24 & SynSaf-P56 & SynSaf-P96 \\
\hline Enterococcus sp. VRE 12204 & 128 & $>128$ & 128 & 64 & 16 \\
\hline Enterococcus sp. VRE 12201 & $>128$ & $>128$ & $>128$ & $>128$ & 128 \\
\hline Enteroccocus sp. VSE 775 & $>128$ & $>128$ & $>128$ & $>128$ & $>128$ \\
\hline S. aureus EMRSA 18 & $>128$ & $>128$ & $>128$ & $>128$ & $>128$ \\
\hline S. aureus EMRSA 15 & $>128$ & $>128$ & $>128$ & $>128$ & 128 \\
\hline S. aureus NCTC 9144 (MSSA) & 128 & 128 & 16 & 32 & 8 \\
\hline
\end{tabular}

Table S-2. Minimal Inhibitory Concentration (MIC) values for the SynSaf-P96 series in $\mu \mathrm{g} / \mathrm{mL}$ for a panel of Gram-positive organisms. MIC is determined as the lowest concentration of peptide needed to prevent overnight growth. 


\begin{tabular}{|c|c|c|}
\hline \multicolumn{3}{|c|}{ MIC $(\mu \mathrm{g} / \mathrm{ml})$} \\
\hline Strain & SynSaf-P56 & SynSaf-P96 \\
\hline E.coli 319238 & 16 & 4 \\
E.coli LEC 001 & 16 & 8 \\
E.coli CFI_161_NDM & 4 & 1 \\
E.coli CFI_033_NDM1 & 8 & 2 \\
E.coli CFI_017_VIM4 & 8 & 2 \\
A. baylii ADP1 & 4 & 1 \\
A. baumannii NCTC 13302 & 2 & 2 \\
A. baumannii A2 & 2 & 1 \\
A. baumannii A7 & 4 & 1 \\
A. baumannii W1 & 2 & 1 \\
A. baumannii NCTC 13424 & 16 & 2 \\
A. baumannii A118 & 2 & 1 \\
\hline
\end{tabular}

Table S-3. Minimal Inhibitory Concentration (MIC) values for SynSaf-P56 and SynSaf-P96 in $\mu \mathrm{g} / \mathrm{mL}$ for an extended panel of multi-drug resistant Gram-negative bacteria. MIC is determined as the lowest concentration of peptide needed to prevent overnight growth 


\begin{tabular}{|c|c|c|c|c|c|c|}
\hline $\begin{array}{c}\text { Syn-safencin } \\
\text { variant }\end{array}$ & Parameter & Level 1 & Level 2 & Level 3 & Level 4 & Level 5 \\
\hline SynSaf-P1 & \multirow{5}{*}{ Amplitude (pA) } & $.386 \pm .049$ & - & - & - & - \\
\hline SynSaf-P8 & & $.378 \pm .021$ & $.997 \pm .109$ & - & - & - \\
\hline SynSaf-P24 & & $.379 \pm .080$ & $1.16 \pm .067$ & $1.67 \pm .024$ & - & - \\
\hline SynSaf-P56 & & $1.034 \pm .164$ & $2.510 \pm .182$ & $4.715 \pm .253$ & $7.253 \pm .226$ & - \\
\hline SynSaf-P96 & & $.711 \pm .080$ & $3.141 \pm .512$ & $6.179 \pm .069$ & $11.020 \pm .768$ & $15.367 \pm 1.688$ \\
\hline SynSaf-P1 & \multirow{5}{*}{$\begin{array}{c}\text { Conductance } \\
\text { (pS) }\end{array}$} & $7.720 \pm .970$ & - & - & - & - \\
\hline SynSaf-P8 & & $7.508 \pm .414$ & $19.943 \pm 2.17$ & - & - & - \\
\hline SynSaf-P24 & & $7.59 \pm 1.59$ & $23.27 \pm 1.35$ & $33.37 \pm .49$ & - & - \\
\hline SynSaf-P56 & & $20.686 \pm 3.263$ & $50.203 \pm 3.646$ & $94.300 \pm 5.061$ & $145.075 \pm 4.527$ & - \\
\hline SynSaf-P96 & & $14.219 \pm 1.603$ & $62.819 \pm 10.233$ & $123.578 \pm 1.374$ & $220.399 \pm 15.366$ & $307.335 \pm 33.764$ \\
\hline SynSaf-P1 & \multirow{5}{*}{$\begin{array}{c}\text { Estimated pore } \\
\text { radius }(\mathrm{nm})\end{array}$} & $.050 \pm .003$ & - & - & - & - \\
\hline SynSaf-P8 & & $.049 \pm .001$ & $.080 \pm .004$ & - & - & - \\
\hline SynSaf-P24 & & $.049 \pm .005$ & $.087 \pm .002$ & $.104 \pm .001$ & - & - \\
\hline SynSaf-P56 & & $.082 \pm .007$ & $.129 \pm .005$ & $.178 \pm .005$ & $.222 \pm .004$ & - \\
\hline SynSaf-P96 & & $.067 \pm .004$ & $.144 \pm .012$ & $.205 \pm .001$ & $.276 \pm .010$ & $.328 \pm .019$ \\
\hline
\end{tabular}

Table S-4. Pore calculations from patch clamp analysis of SynSaf-P96 series. Data is representative of three traces $(n=3)$. Calculations were done as described in the materials and methods. +/- indicates the SEM. 


\begin{tabular}{|c|c|c|}
\hline \multicolumn{3}{|c|}{$\mathrm{FICl}$ values } \\
\hline SynSaf-P & A. baumanii AYE & E. coli NCTC 12923 \\
\hline $1 \& 8$ & 2 & 1 \\
\hline $1 \& 24$ & 2 & 0.5 \\
\hline $1 \& 56$ & 1 & 0.5 \\
\hline $1 \& 96$ & 0.5 & 0.5 \\
\hline $8 \& 24$ & 0.5 & 0.5 \\
\hline $8 \& 56$ & 0.5 & 1 \\
\hline $8 \& 96$ & 0.25 & 0.5 \\
\hline $24 \& 56$ & 1 & 0.5 \\
\hline $24 \& 96$ & 0.25 & 0.5 \\
\hline $56 \& 96$ & 0.5 & 1 \\
\hline
\end{tabular}

Table S-5. Synergy is detectable between non-membrane disrupting (SynSaf-P1 and SynSaf-P8) and membrane disrupting antimicrobial peptides (SynSaf-P96). FICI values for peptide-peptide interactions within the Syn-SafP96 series. Synergy is defined as an FICI $\leq .5$. 


\begin{tabular}{|c|c|c|c|c|c|c|}
\hline SynSaf-P & Parameter & Level 1 & Level 2 & Level 3 & Level 4 & Level 5 \\
\hline SynSaf-P8 & \multirow{3}{*}{ Amplitude (pA) } & - & - & - & - & - \\
\hline SynSaf-P96 & & $.612 \pm .021$ & $1.395 \pm .109$ & - & - & - \\
\hline SynSaf-P8 \& 96 & & $.672 \pm .034$ & $2.353 \pm .098$ & $4.163 \pm .151$ & $6.366 \pm .363$ & $8.919 \pm .194$ \\
\hline SynSaf-P8 & \multirow{3}{*}{ Conductance (pS) } & - & & - & - & - \\
\hline SynSaf-P96 & & $12.234 \pm 3.751$ & $27.909 \pm 2.346$ & - & - & - \\
\hline SynSaf-P8 \& 96 & & $13.437 \pm .687$ & $47.052 \pm 1.961$ & $83.258 \pm 3.014$ & $127.322 \pm 7.264$ & $178.389 \pm 3.871$ \\
\hline SynSaf-P8 & \multirow{3}{*}{$\begin{array}{l}\text { Estimated pore radius } \\
\qquad(\mathrm{nm})\end{array}$} & - & - & - & - & - \\
\hline SynSaf-P96 & & $.061 \pm .010$ & $.0956 \pm .004$ & - & - & - \\
\hline SynSaf-P8 \& 96 & & $.066 \pm .002$ & $.125 \pm .003$ & $.167 \pm .003$ & $.208 \pm .006$ & $.247 \pm .003$ \\
\hline
\end{tabular}

Table S-6. Pore calculations from patch clamp detection of peptide synergy. Data is representative of three traces $(n=3)$. Calculations were done as described in the materials and methods. $+/$ - indicates the SEM. 


\begin{tabular}{|c|c|c|}
\hline \multicolumn{3}{|c|}{ Peptide Toxicity on G. mellonella } \\
\hline Treatment & Concentration & $\%$ Survival \\
\hline SynSaf-P1 & $150 \mathrm{mg} / \mathrm{kg}$ & 90 \\
SynSaf-P8 & $150 \mathrm{mg} / \mathrm{kg}$ & 100 \\
SynSaf-P24 & $150 \mathrm{mg} / \mathrm{kg}$ & 100 \\
SynSaf-P56 & $150 \mathrm{mg} / \mathrm{kg}$ & 100 \\
SynSaf-P96 & $150 \mathrm{mg} / \mathrm{kg}$ & 100 \\
SynSaf-P96 + SynSaf-P8 & $75 \mathrm{mg} / \mathrm{kg}(37.5 \mathrm{mg} / \mathrm{kg}$ of each) & 100 \\
Meropenem & $60 \mathrm{mg} / \mathrm{kg}$ & 100 \\
PBS & $\mathrm{N} / \mathrm{A}$ & 100 \\
\hline
\end{tabular}

Table S-7. In vivo toxicity of the syn-safencin peptides in G. mellonella. All compounds were administered as a single $10 \mu \mathrm{L}$ injection. Percent survival was assessed after three days. Each group consisted of 10 caterpillars $(n=10)$. 